\title{
Dimensionamento de pilares compostos por tubos de aço preenchidos com concreto em situação de incêndio
}

\author{
Fábio Masini Rodrigues ${ }^{1 *}$ e Armando Lopes Moreno Júnior ${ }^{2}$ \\ ${ }^{1}$ Faculdade de Engenharia Civil e Arquitetura, Universidade Estadual de \\ Campinas, fabiosecfmr@gmail.com \\ ${ }^{2}$ Faculdade de Engenharia Civil e Arquitetura, Universidade Estadual de \\ Campinas, almoreno@fec.unicamp.br
}

\section{Fire design of concrete-filled steel tube composite columns}

\begin{abstract}
Resumo
Os pilares compostos por tubos preenchidos com concreto trazem vantagens em construções residenciais e industriais devido ao seu desempenho estrutural, rapidez e facilidade de execução. Nesse contexto, com a utilização do software $A B A Q U S$, foram elaboradas tabelas para definir temperaturas na seção transversal, condição inicial para o dimensionamento dos pilares em situação de incêndio. $O$ dimensionamento dos pilares, através do procedimento descrito no Eurocode 4, foi apresentado e comparado com outros procedimentos analíticos simplificados e, também, comparado com os resultados obtidos por meio de análise numérica. Os resultados do presente estudo mostraram que o dimensionamento dos pilares, através das tabelas com as temperaturas na seção transversal e processo indicado no Eurocode 4, são satisfatórios para pilares com tubos de diâmetros e dimensões da seção transversal reduzidos, fora dos limites indicados pelo Eurocode 4.
\end{abstract}

Palavras-chave: pilar misto; dimensionamento; incêndio

\begin{abstract}
The composed columns of steel tubes filled with concrete show a high structural performance in industrial and residential constructions, besides the quickness of execution. In this context, with the use of ABAQUS software, tables were elaborated to define temperatures in the cross section, initial condition for the design of the columns in a fire situation. The design of the columns through the procedure described in Eurocode 4 was presented and compared with other simplified analytical procedures and also compared with the results obtained by means of numerical analysis. The results of the present study showed that the design of the columns through the tables with the temperatures in cross section and simplified process indicated in Eurocode 4 are satisfactory for columns with small tubes, outside the limits indicated by Eurocode 4.
\end{abstract}

Keywords: composite column; structural design; fire

*Autor correspondente 


\section{Introdução}

Os pilares mistos compostos por tubos de aço preenchidos com concreto apresentam vantagens com relação aos pilares de aço, do ponto de vista estético, construtivo e estrutural em situação de incêndio e, em construções residenciais e industriais de poucos pavimentos, são normalmente utilizados pilares mistos, com tubos de aço de menores dimensões de seção transversal.

A exigência de resistência ao fogo para elementos estruturais e elementos componentes do sistema construtivo é estabelecida, pelas normas nacionais e internacionais, por meio do TRRF (Tempo Requerido de Resistência ao Fogo), que são preestabelecidos entre 30 e 120 minutos, com intervalos de 30 minutos.

Com relação à verificação em situação de incêndio, a norma ABNT NBR 14432:2001 isenta as estruturas de edificações residenciais térreas, edificações com área construída inferior a $750 \mathrm{~m}^{2}$ e edificações com dois pavimentos cuja área total seja inferior a 1500 $\mathrm{m}^{2}$ e com carga de incêndio não superior a $1000 \mathrm{MJ} / \mathrm{m}^{2}$. Contudo, uma edificação com maior área e adequadamente compartimentada é menos vulnerável aos efeitos de um incêndio, do que uma edificação de menor área sem uma efetiva compartimentação (Silva, 2003).

Nesse contexto, os pilares mistos compostos por tubos preenchidos com concreto e sem adição de barras de aço, podem ser uma alternativa técnica e economicamente vantajosa. No entanto, esses pilares de menor dimensão de seção transversal, normalmente, ficam fora do campo de aplicação dos métodos analíticos simplificados, indicados no Eurocode 4.

No presente artigo, visando oferecer uma abordagem prática para o dimensionamento de pilares mistos com tubos de seção transversal quadrado e circular de pequenas dimensões, em situação de incêndio, foram elaboradas tabelas, por meio de modelos numéricos, às quais indicam as temperaturas em camadas ao longo da seção transversal de pilares mistos. Também foram apresentadas tabelas semelhantes, elaboradas por Renaud (2004).

As tabelas propostas no presente trabalho foram elaboradas considerando os tubos de aço comercializados no Brasil. 
As respostas, obtidas com a utilização das tabelas com os campos de temperaturas e dos processos simplificados, serão confrontadas, com base nas respostas de modelos numéricos tridimensionais, elaborados por meio do software ABAQUS.

\section{Materiais e método}

\subsection{Sequência metodológica}

No presente trabalho, foram elaborados modelos numéricos planos de pilares mistos compostos por tubos de aço de seção quadrada e circular com pequenas dimensões, cuja seção transversal fora subdividida em camadas. As temperaturas representativas de cada camada foram transcritas e organizadas em tabelas práticas, cujos valores foram comparados aos indicados nas tabelas elaboradas por Renaud (2004). Considerou-se a temperatura representativa para uma determinada camada, a média das temperaturas nodais (nós dos elementos finitos) pertencentes à respectiva camada.

O procedimento simplificado de dimensionamento, método geral do Eurocode 4 , foi utilizado para determinar a normal última em situação de incêndio de cada pilar analisado e essa, foi comparada à normal última obtida por meio de modelos numéricos tridimensionais.

As temperaturas tomadas diretamente dos modelos numéricos foram comparadas às determinadas por meio de equações simplificadas indicadas em Rodrigues e Moreno Jr. (2017) para pilares mistos de seção quadrada e em Espinós (2012) para pilares mistos de seção circular.

\subsection{Características dos exemplares nos modelos numéricos e ação térmica}

Os pilares escolhidos para o presente estudo estão indicados na Figura 1.

\begin{tabular}{|c|c|c|c|}
\hline Denominação & Seção & $\begin{array}{c}\text { Dimensão do tubo } \\
\text { b ou d (mm) }\end{array}$ & $\begin{array}{c}\text { Espessura do tubo } \\
\mathbf{t}(\mathbf{m m})\end{array}$ \\
\hline $\mathrm{PQ}-100-5.2$ & Quadrado & 100.0 & 5.2 \\
\hline $\mathrm{PQ}-120-5$ & Quadrado & 120.0 & 5.0 \\
\hline $\mathrm{PQ}-140-5.6$ & Quadrado & 140.0 & 5.6 \\
\hline $\mathrm{PQ}-160-6.4$ & Quadrado & 160.0 & 6.4 \\
\hline $\mathrm{PQ}-200-6.4$ & Quadrado & 200.0 & 6.4 \\
\hline $\mathrm{PC}-114.3-4$ & Circular & 114.3 & 4.0 \\
\hline $\mathrm{PC}-141.3-5.6$ & Circular & 141.3 & 5.6 \\
\hline $\mathrm{PC}-168.3-6.4$ & Circular & 168.3 & 6.4 \\
\hline $\mathrm{PC}-219.1-8$ & Circular & 219.1 & 8.0 \\
\hline
\end{tabular}

Figura 1 - Características dos pilares mistos 
$\mathrm{O}$ aço do tubo foi considerado com resistência ao escoamento de $350 \mathrm{MPa}$ e o concreto com resistência à compressão de $30 \mathrm{MPa}$.

A ação térmica foi aplicada no entorno dos pilares, agindo uniformemente ao longo de todo o elemento. Foi adotada a curva de incêndio padrão (ISO 834) e no modelo numérico foi considerado que, os gases no entorno do elemento estrutural são aquecidos por radiação e convecção que, consequentemente, aquecem a face externa do elemento e, por radiação, convecção e condução, é estabelecido o campo de temperaturas em todo o elemento estrutural. Nos modelos planos, os campos de temperaturas de interesse foram obtidos para 30, 60 e 90 minutos de exposição ao fogo.

\subsection{Modelos numéricos planos (análise de transferência de calor)}

Nos modelos foram considerados os seguintes parâmetros: elemento finito quadrilateral DC2D4, para os pilares com seção quadrada; elemento triangular DC2D3, para os pilares de seção circular; temperatura inicial definida em $20^{\circ} \mathrm{C}$; fator de radiação e de emissividade do fogo igual a 1.0 e fator da face exposta do tubo de 0.7; coeficiente de convecção para superfície exposta de $25 \mathrm{~W} / \mathrm{m}^{2}{ }^{\circ} \mathrm{C}$ e constante de Stefan-Boltzmann de $5.67 \times 10^{-8} \mathrm{Wm}^{-2} \mathrm{~K}^{-4}$; densidade do aço considerada com o valor constante de 7850 $\mathrm{kg} / \mathrm{m}^{3}$ e do concreto, com o valor constante de $2300 \mathrm{~kg} / \mathrm{m}^{3}$; umidade do concreto adotado com 3\%; nos modelos planos a resistência térmica na interface entre o tubo de aço e o núcleo concreto foi negligenciada (contato térmico perfeito); foi adotado o limite superior da condutividade térmica do concreto; demais propriedades térmicas foram adotadas conforme Eurocode 4.

Segue na Figura 2 o campo de temperaturas para 60 minutos de exposição ao fogo.
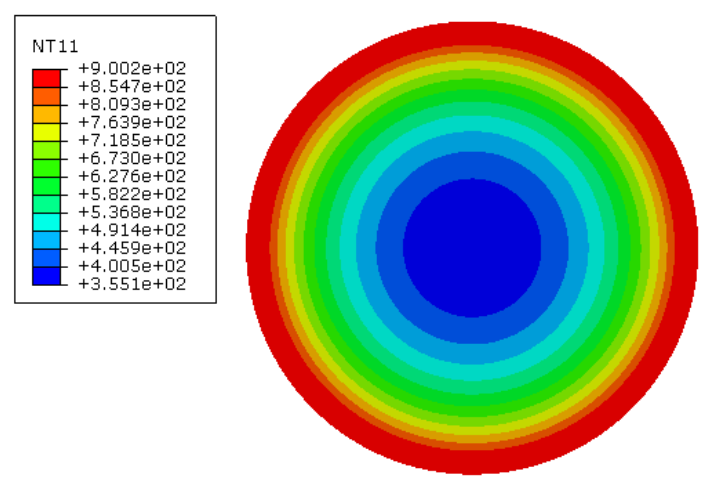

Figura 2 - Campo de temperaturas para o exemplar PC-168.3-6.4 


\subsection{Modelos tridimensionais (análise termomecânica)}

Os modelos tridimensionais foram elaborados para os exemplares: PC-114.3-4, PC168.3-6.4, PQ-100-5.2 e PQ-140-5.6, todos com um comprimento longitudinal de $3.5 \mathrm{~m}$, correspondente a um comprimento de flambagem em situação de incêndio de $1.75 \mathrm{~m}$ $\left(L_{f l, \theta}=0.5 \times L\right)$, conforme processo simplificado. Nos modelos tridimensionais foi considerada a não linearidade com uma imperfeição geométrica inicial equivalente a 1/500 do comprimento do tubo, conforme Dotreppe (2007).

A força normal foi aplicada nos modelos tridimensionais de forma centrada, sua intensidade foi definida pelo método simplificado, com o objetivo de comparar o tempo de resistência ao fogo determinado por ambos os métodos, simplificado e avançado. A força normal foi aplicada em um ponto de referência (RP2), acoplado à seção da extremidade superior (topo) do pilar, tendo sido associado ao mesmo, um vínculo externo articulado e com liberdade à translação na direção vertical (z). Também foi adicionado um ponto de referências (RP1), acoplado à seção da base do pilar e, a esse, foi associado um vínculo articulado, com restrição à translação nas 3 direções ortogonais $(x, y$ e $z)$

Na Figura 3 estão representados os vínculos definidos nos modelos.

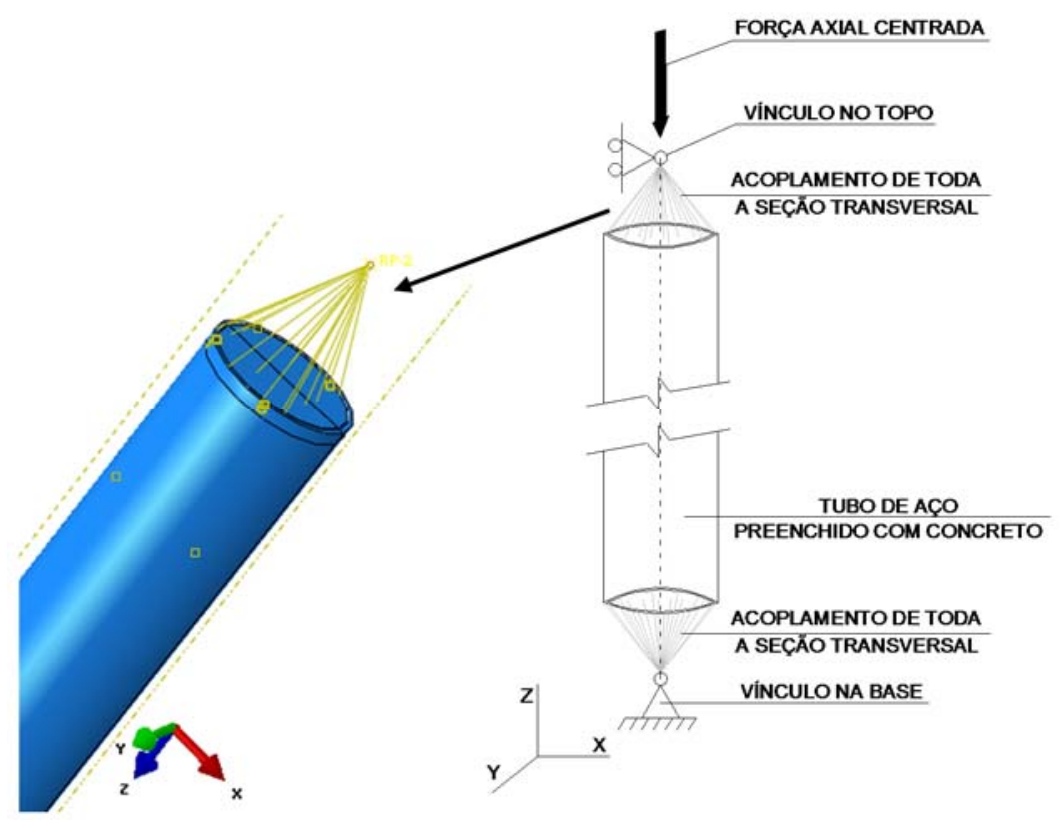

Figura 3 - Características dos vínculos nos modelos numéricos 
Foi considerada para os modelos tridimensionais, a análise conjunta com o solver explicit, com iteração entre as análises, térmica e mecânica. A força axial foi aplicada inicialmente (step 1) e, em seguida (step 2), o elemento foi aquecido, até que o mesmo esgote sua capacidade resistente. A resistência térmica à condução entre o tubo de aço e o núcleo de concreto foi considerado pelo software e calibrado para um valor médio de $0.02 \mathrm{~m}^{2} \mathrm{~K} / \mathrm{W}$, conforme indicado em Espinós (2012).

Para considerar o esgotamento da capacidade resistente do elemento estrutural, foi adotado o critério da norma EN 1363-1, cuja falha é caracterizada pela contração axial máxima de $1 \%$ do comprimento do pilar e pela taxa de contração axial de $0.3 \%$ do comprimento do pilar por minuto;

Como definições específicas para a análise termomecânica, pode-se citar: hard contact e o penalty contact com coeficiente de atrito constante de 0.3 , definidos para o contato mecânico normal e tangencial entre o tubo de aço e o núcleo de concreto; módulo de elasticidade do concreto e o do aço, conforme equações constitutivas apresentadas pelo Eurocode 4, assim como o comportamento plástico dos materiais, que foi determinado pelos valores de tensão versus deformação, variando com a temperatura; concreto definido conforme o modelo CDP (Concrete Damage Plasticity), com os parâmetros indicados em Rodrigues (2012) sendo: $\psi=35^{\circ}, \sigma_{\mathrm{bo}} / \sigma_{\mathrm{c} 0}=1.16, \mathrm{~m}=0.1, \mathrm{~K}=0.667$ e $\mu=0$.

Seguem na Figura 4 os deslocamentos axiais do pilar com seção quadrada.

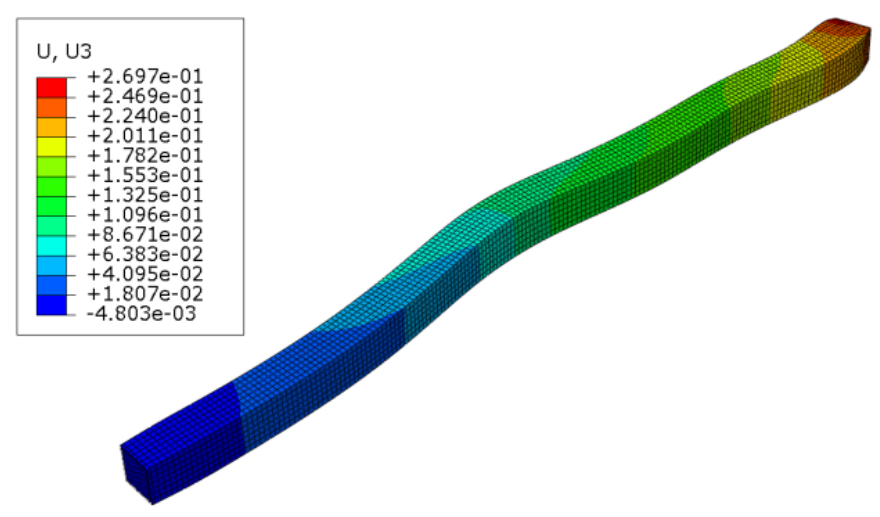

Figura 4 - Deslocamento axial do exemplar PQ-140-5.6 


\subsection{Procedimentos analíticos}

\subsubsection{Eurocode 4}

O Eurocode 4 apresenta procedimentos analíticos simplificados para dimensionamento de pilares mistos em situação de incêndio, um para pilares compostos por tubos de aço preenchidos com concreto, descrito no anexo $\mathrm{H}$ e cujos resultados se revelaram inseguros, principalmente para pilares com maior esbeltez (Aribert et al, 2008). Outro procedimento é descrito em seu anexo G, para dimensionamento de pilares constituídos de perfis parcialmente revestidos com concreto e, ainda, um método geral.

Para o dimensionamento de um pilar com força axial centrada em situação de incêndio, a força normal de cálculo não deve superar a força normal resistente em situação de incêndio. Dada a probabilidade de ocorrência de um incêndio, os coeficientes de ponderação e majoração da força normal são reduzidos, conforme Eurocode 4 ou ABNT NBR8681:2004.

Os procedimentos analíticos consideram o campo de temperatura estabelecido na seção transversal para um determinado tempo de exposição ao fogo e a respectiva depreciação das propriedades dos materiais.

Para definir a distribuição de temperaturas em pilares mistos com tubo de aço preenchido com concreto é necessário recorrer, por exemplo, à metodologia proposta por LIE e WHITE descrita em RIGAZZO (2006), contudo, de difícil aplicação prática, ou recorrer às tabelas apresentadas no presente trabalho ou em Renaud (2004), ambas elaboradas por meio de simulações numéricas.

O método descrito no anexo $\mathrm{H}$ consiste em determinar a normal última em situação de incêndio, considerando um campo de temperaturas preestabelecido e a depreciação das propriedades dos materiais. 


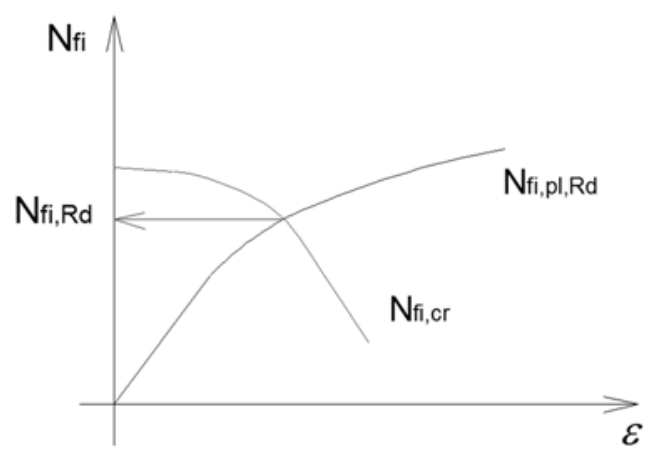

Figura 5 - Curva crítica de Euler e força normal plástica, em situação de incêndio A força normal última é encontrada quando a curva da força normal plástica intercepta a curva que representa a carga crítica Euler (flambagem elástica). Ambas as curvas devem ser construídas com as propriedades dos materiais depreciadas (Figura 5).

Limites para aplicação do procedimento do anexo $\mathrm{H}$, indicados no Eurocode 4: esbeltez relativa máxima de 0,5; comprimento de flambagem de até 4,5 m; diâmetro ou lado menor da seção do tubo entre 140 e 400 mm; resistência do concreto a compressão entre 20 e $40 \mathrm{MPa}$ e, porcentagem de área das barras de aço entre 0\% e 5\%.

O método geral se estendeu para o dimensionamento de pilares com tubos de aço preenchidos com concreto, no entanto, existe um número reduzido de estudos para validação da aplicabilidade do método, conforme mencionam Wang 1997, Renaud et al. 2004 e Aribert et al. 2008.

Limites para aplicação do método: pilares devem ser contraventados; devem apresentar dupla simetria; o coeficiente de contribuição do aço deve estar entre $0,2 \leq \delta \leq 0,9$, sendo $\delta=A_{s} . f_{y d} / N_{p l, R d}$; a resistência ao escoamento do aço deve estar entre 235 e $460 \mathrm{MPa}$; a resistência à compressão do concreto entre 20 e $50 \mathrm{MPa}$; a taxa geométrica de armação do pilar deve ser de no máximo 6\%; o índice de esbeltez relativo de ser igual ou inferior a 2; a relação entre a altura e largura da seção transversal retangulares deve estar entre 0,2 e 5; para seções envolvidas por concreto deve ser disposta armação longitudinal e transversal; as seções preenchidas por concreto podem não conter armação.

O método geral apresenta as Equação 1 a 5, cuja Equação 1 é utilizada para determinar a normal última da seção transversal em situação de incêndio. 
$\mathrm{N}_{\mathrm{fi}, \mathrm{Rd}}=\chi_{\theta} \cdot \mathrm{N}_{\mathrm{fi}, \mathrm{pl}, \mathrm{Rd}}$

Sendo: $\mathrm{N}_{\mathrm{fi}, \mathrm{Rd}}$, a força normal última de cálculo em situação de incêndio; $\chi_{\theta}$, o fator de redução fornecido pela curva de dimensionamento "c" do EN 1993-1-1, em função da esbeltez relativa; $\mathrm{N}_{\mathrm{fi}, \mathrm{pl}, \mathrm{Rd}}$, força normal de plastificação de cálculo em situação de incêndio.

A força normal de plastificação é determinada conforme Equação 2.

$\mathrm{N}_{\mathrm{fi}, \mathrm{pl}, \mathrm{Rd}}=\sum_{\mathrm{j}}\left(\mathrm{A}_{\mathrm{a}} \cdot \mathrm{f}_{\mathrm{a}, \max , \theta}\right)+\sum_{\mathrm{k}}\left(\mathrm{A}_{\mathrm{s}} \cdot \mathrm{f}_{\mathrm{s}, \max , \theta}\right)+\sum_{\mathrm{m}}\left(\mathrm{A}_{\mathrm{c}} \cdot \mathrm{f}_{\mathrm{c}, \max , \theta}\right)$

Sendo: $\sum_{\mathrm{j}}\left(\mathrm{A}_{\mathrm{a}} \cdot \mathrm{f}_{\mathrm{a}, \max , \theta}\right)$, o somatório do produto da área da seção do tubo de aço pela resistência do aço, em situação de incêndio; $\sum_{\mathrm{k}}\left(\mathrm{A}_{\mathrm{s}} \cdot \mathrm{f}_{\mathrm{s}, \mathrm{max}, \theta}\right)$, o somatório dos produtos da área das barras da armadura pela resistência ao escoamento do aço, em situação de incêndio; $\sum_{\mathrm{m}}\left(\mathrm{A}_{\mathrm{c}} \cdot \mathrm{f}_{\mathrm{c}, \mathrm{max}, \theta}\right)$, o somatório dos produtos dos elementos de área do concreto pela resistência característica à compressão, em situação de incêndio.

O índice de esbeltez relativo é determinado pela Equação 3.

$\bar{\lambda}_{, \theta}=\sqrt{\frac{\mathrm{N}_{\mathrm{fi}, \mathrm{pl}, \mathrm{Rd}}}{\mathrm{N}_{\mathrm{fi}, \mathrm{cr}}}}$

Onde: $\mathrm{N}_{\text {fi,cr }}$ é a carga crítica de Euler em situação de incêndio, dada pela Equação 4.

$N_{f i, c r}=\frac{\pi^{2} \cdot(E I)_{, e f f, \theta}}{L_{\theta}^{2}}$

Sendo: (EI),eff, $\theta$, o produto do módulo de elasticidade pela inércia da seção do pilar misto à flexão em situação de incêndio, dado pela Equação 5.

$(\mathrm{EI})_{, \mathrm{eff}, \theta}=\sum_{\mathrm{j}}\left(\varphi_{\mathrm{a}, \theta} \cdot \overline{\mathrm{E}}_{\mathrm{a}, \theta} \cdot \mathrm{I}_{\mathrm{a}}\right)+\sum_{\mathrm{k}}\left(\varphi_{\mathrm{s}, \theta} \cdot \overline{\mathrm{E}}_{\mathrm{s}, \theta} \cdot \mathrm{I}_{\mathrm{s}}\right)+\sum_{\mathrm{m}}\left(\varphi_{\mathrm{c}, \theta} \cdot \overline{\mathrm{E}}_{\mathrm{c}, \mathrm{sec}, \theta} \cdot \mathrm{I}_{\mathrm{c}}\right)$

Sendo:

$\overline{\mathrm{E}}_{\mathrm{a}, \theta}, \overline{\mathrm{E}}_{\mathrm{s}, \theta}, \overline{\mathrm{E}}_{\mathrm{c}, \mathrm{sec}, \theta}$ : módulo de deformação longitudinal do aço do perfil, das barras de reforço e do concreto; $\mathrm{I}_{\mathrm{a}}, \mathrm{I}_{\mathrm{s}}, \mathrm{I}_{\mathrm{c}}$ : momento de inércia da seção do perfil de aço, das barras de reforço e do concreto; $\varphi_{\mathrm{a}, \theta}, \varphi_{\mathrm{s}, \theta}, \varphi_{\mathrm{c}, \theta}$ : coeficiente de redução que depende dos efeitos das tensões térmicas no perfil de aço, nas barras de reforço e no concreto, conforme apresentados na Tabela 1 em função do TRRF e do material.

Tabela 1 - Coeficientes de redução (Fonte: Adaptado do Eurocode 4)

\begin{tabular}{|c|c|c|c|}
\hline $\begin{array}{c}\text { TRRF } \\
\text { (minutos) }\end{array}$ & $\begin{array}{c}\text { Perfil de aço } \\
\boldsymbol{\varphi}_{\mathrm{a}, \theta}\end{array}$ & $\begin{array}{c}\text { Armadura } \\
\boldsymbol{\varphi}_{\mathrm{s}, \boldsymbol{\theta}}\end{array}$ & $\begin{array}{c}\text { Concreto } \\
\boldsymbol{\varphi}_{\mathrm{c}, \boldsymbol{\theta}}\end{array}$ \\
\hline 30 & 1.0 & 1.0 & 0.8 \\
\hline 60 & 0.9 & 0.9 & 0.8 \\
\hline 90 & 0.8 & 0.8 & 0.8 \\
\hline 120 & 1.0 & 1.0 & 0.8 \\
\hline
\end{tabular}


O coeficiente $\varphi_{c \theta}$ indicado com o valor de 0,8 deve ser utilizado quando o módulo de elasticidade for determinado por $\mathrm{E}_{\mathrm{ci}}=3 / 2 \mathrm{E}_{\mathrm{cm}}$, sendo $\mathrm{E}_{\mathrm{cm}}$ o módulo de elasticidade obtido a partir da resistência média do concreto à compressão, sendo: $E_{c m}=$ $22\left(\frac{f c+8}{10}\right)^{0,3}$. O módulo de elasticidade do concreto em elevadas temperaturas é determinado depreciando o módulo de elasticidade em temperatura ambiente pelo fator $K E_{c, \theta}$ definido na Equação 6.

$\mathrm{K}_{\mathrm{Ec}, \theta}=\mathrm{Kc}_{, \theta} \cdot \frac{\varepsilon_{\mathrm{cu}}}{\varepsilon_{\mathrm{cu}, \theta}}$

Sendo: $\mathrm{K}_{\mathrm{Ec}, \theta}$, fator de redução do módulo de elasticidade do concreto; $\mathrm{K}_{\mathrm{c}, \theta}$, fator de redução da resistência do concreto a compressão; $\varepsilon_{c u}$, deformação última do concreto em temperatura ambiente; $\varepsilon_{\mathrm{cu}, \theta}$, deformação última do concreto em elevada temperatura.

O fator de redução da resistência do concreto à compressão, a deformação última do concreto em temperatura ambiente e em elevada temperatura são indicados no Eurocode 4.

Hager \& Krzemien (2015) avaliou, com base em ensaios experimentais, o módulo de elasticidade do concreto a elevadas temperaturas, considerando concretos de normal e alta resistência, além de considerar a variação de umidade e dos tipos de agregados e concluiu que o coeficiente de depreciação do módulo de elasticidade do concreto, definido conforme Eurocode 4, fornece valores conservadores para concretos de resistência normal e inseguros para concretos de alta resistência, os autores também indicam as Equações 7 como proposta para concretos de normal e alta resistência.

$K E_{c, \theta}=\frac{E_{c, \theta}}{E_{c}} \mathrm{Com} \mathrm{E}_{\mathrm{c}, \theta}=\left\{\begin{array}{l}\operatorname{Ec}\left(20^{\circ} \mathrm{C}\right) \times(1,067-0,0033 \times \theta) \mathrm{p} / 20^{\circ} \mathrm{C} \leq \theta \leq 200^{\circ} \mathrm{C} \\ \operatorname{Ec}\left(20^{\circ} \mathrm{C}\right) \times(0,6-0,001 \times \theta) \mathrm{p} / 200^{\circ} \mathrm{C}<\theta \leq 600^{\circ} \mathrm{C} \\ 0 \mathrm{p} / 600^{\circ} \mathrm{C}<\theta\end{array}\right.$

Segue na Figura 6 o gráfico com os valores dos fatores $K E_{c, \theta}$ determinados conforme Eurocode 4 para concreto silicoso e conforme Hager \& Krzemien (2015). 


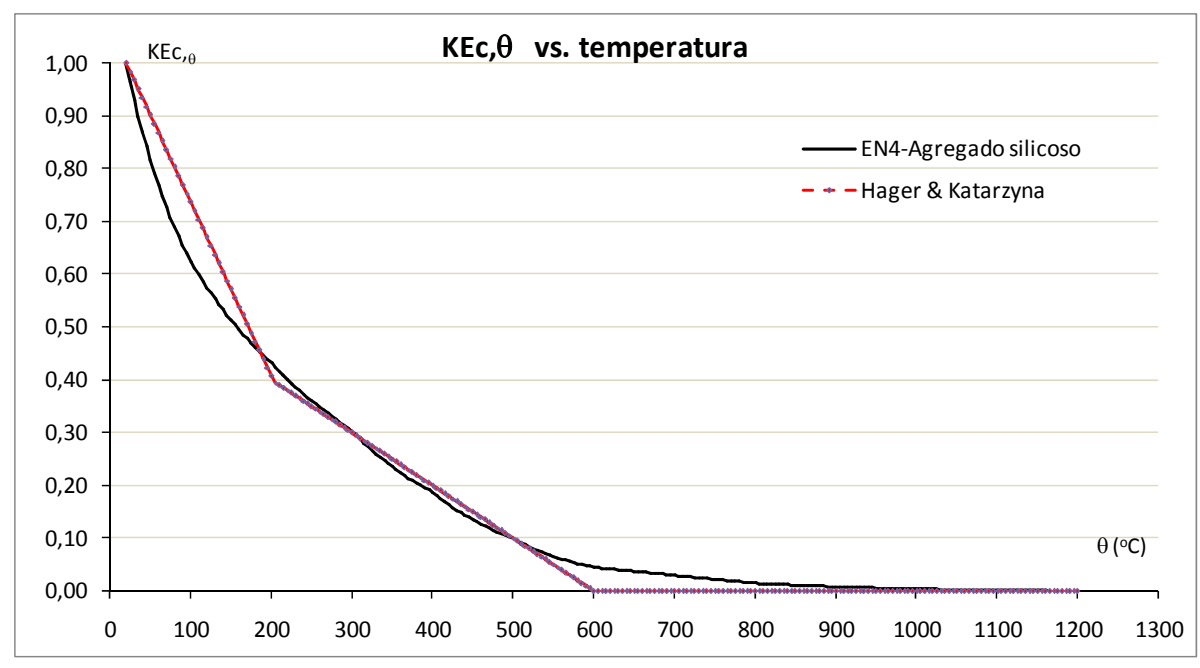

Figura 6 - Coeficiente de redução do módulo de elasticidade do concreto

O comprimento de flambagem em situação de incêndio $\left(L_{\theta}\right)$ pode ser tomado igual ao comprimento do pilar, multiplicado pelos coeficientes de 0,5 para pilares em níveis intermediários e 0,7 para pilares no último lance.

Conforme EN 1993-1-1, as curvas de dimensionamento são apresentadas como curvas "a, b, c, d e ao" (Figura 7). O Eurocode 4 indica a curva de dimensionamento "c" para pilares misto com perfil tubular preenchido com concreto em situação de incêndio. 0 procedimento simplificado indicado em Espinós (2012) indica a curva "a" com os coeficientes $\varphi_{\mathrm{a}, \theta}, \varphi_{\mathrm{s}, \theta}, \varphi_{\mathrm{c}, \theta}$ ajustados pela Autora.

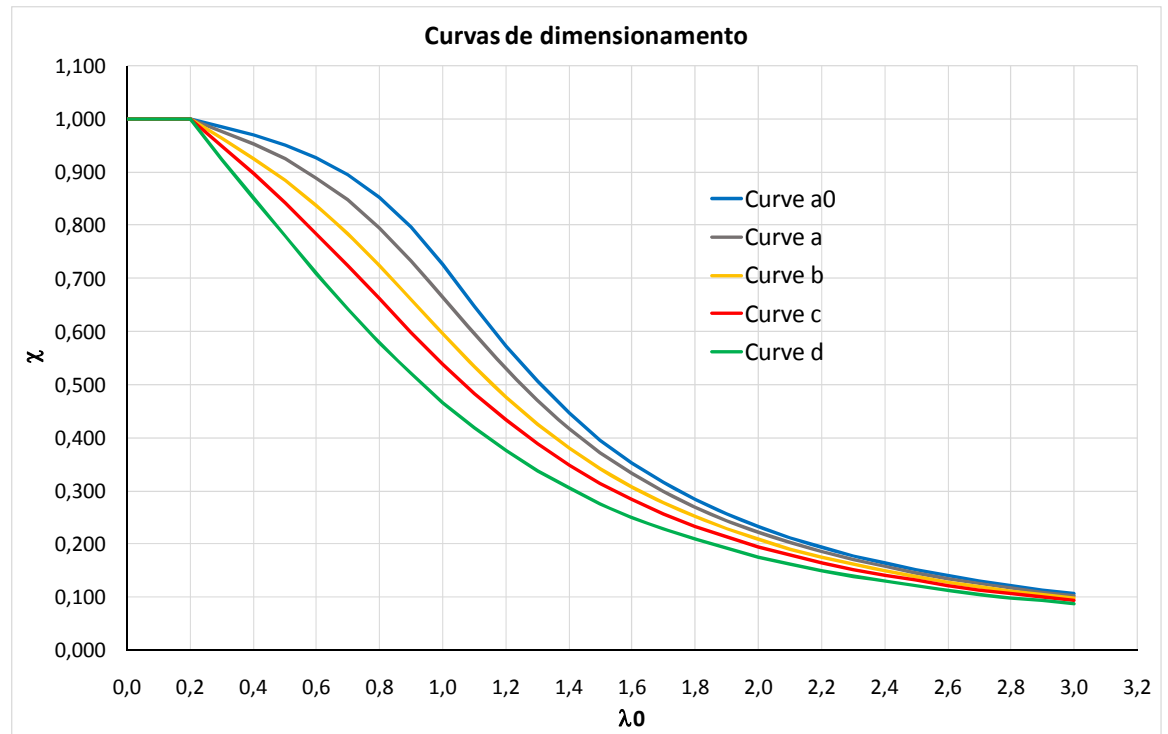

Figura 7 - Curvas de dimensionamento segundo o Eurocode 3 


\subsubsection{Processos analíticos simplificados}

O dimensionamento dos pilares misto é realizado conforme o método geral, considerando um prévio conhecimento do campo de temperaturas na seção transversal a um dado tempo de exposição ao fogo de interesse, normalmente 30, 60, 90 ou 120 minutos.

As temperaturas na seção transversal para os pilares indicados na Figura 1 podem ser obtidas através das tabelas da Figura 9, elaboradas pelo autor. Para os pilares com outras dimensões podem ser utilizadas as tabelas da Figura 10 (Renaud, 2004), sendo válida a interpolação das temperaturas tabeladas para pilares com dimensões intermediárias.

Outro modo de definir temperaturas na seção transversal seria por meio de equações simplificadas, em Rodrigues e Moreno Jr. (2017), por exemplo, são apresentadas as Equações 8 e 9 para pilares com seção quadrada e, em Espinós (2012), são apresentadas as Equações 11 e 12 para pilares com seção circular.

$$
\begin{aligned}
\theta c, e q= & {\left[\left(2,9892 \cdot R^{3}-605 \cdot R^{2}+29982 \cdot R-374314\right) \cdot\left(\frac{u}{A}\right)^{2}\right]+} \\
& {\left[\left(-0,1723 \cdot R^{3}+33,45 \cdot R^{2}-1495 \cdot R+24399\right) \cdot\left(\frac{u}{A}\right)\right]+}
\end{aligned}
$$

$2,643 \cdot 10^{-3} \cdot R^{3}-0,545838 \cdot R^{2}+33,21 \cdot R-317$

$$
\begin{aligned}
\theta a, \text { eq }= & {\left[\left(-2,2 \cdot 10^{-3} \cdot R^{3}+0,6393 \cdot R^{2}-67 \cdot R+4023\right) \cdot\left(\frac{u}{A}\right)\right] } \\
& +7,17 \cdot 10^{-4} \cdot R^{3}-0,2127 \cdot R^{2}+22,54 \cdot R+108
\end{aligned}
$$

Por meio das equações simplificadas são obtidas temperaturas equivalentes, ou seja, são temperaturas médias equivalentes, para o tubo de aço e para todo o núcleo de concreto.

$$
\begin{aligned}
& \theta_{a, e q}=342,1+10,77 R-0,044 R^{2}+3,922 \frac{u}{A}-0,025 R \frac{u}{A} \\
& \theta_{c, \text { eq }}=-186,44+5,764 R-0,026 R^{2}+22,577 \frac{u}{A}-0,032 R\left(\frac{u}{A}\right)^{2}+0,14 R \frac{u}{A}
\end{aligned}
$$

Sendo: $\theta_{\mathrm{a}, \mathrm{eq}}$, a temperatura equivalente do tubo de aço $\left({ }^{\circ} \mathrm{C}\right) ; \theta_{\mathrm{c}, \mathrm{eq}}$, a temperatura equivalente do núcleo de concreto $\left({ }^{\circ} \mathrm{C}\right) ; \mathrm{R}$, o tempo de duração do fogo (min.); u , o perímetro da seção transversal; A , a área da seção transversal. 
Para as Equações 8 e 9 o perímetro e área da seção devem ser em $\mathrm{mm}$ e $\mathrm{mm}^{2}$ e para as Equações 10 e 11 em $\mathrm{m}$ e $\mathrm{m}^{2}$.

\subsubsection{Utilização das tabelas para definir o campo de temperatura}

As temperaturas representativas de cada camada concêntrica (Figura 8) referem-se às temperaturas médias em cada camada, obtidas pelo somatório das temperaturas tomadas nos nós dos elementos finitos, dividido pela quantidade de nós da respectiva camada. As tabelas das Figuras 9 e 10 indicam as temperaturas nas camadas localizadas pela relação b/bi ou d/di, para 30 e 60 minutos de TRRF, conforme ABNT NBR 14432:2001, considerando os tipos de edificações descritas no presente trabalho.

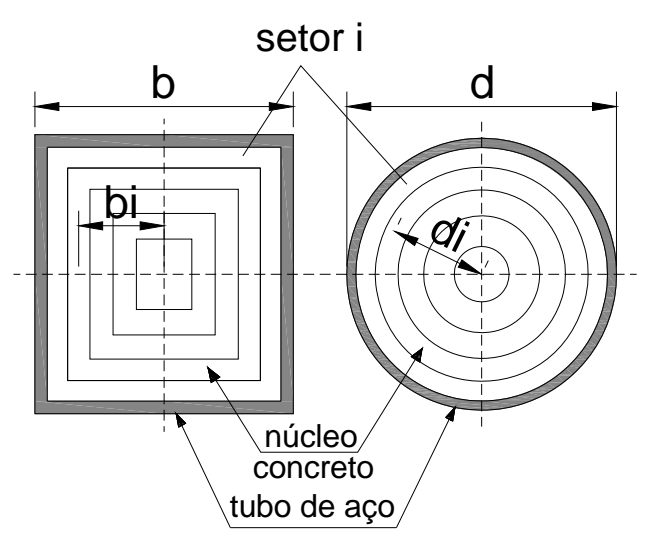

Figura 8 - Divisão da seção transversal

\section{Resultados e discussões}

\subsection{Temperaturas ao longo da seção transversal}

As temperaturas indicados por Renaud (2004) foram definidas por meio de modelos numéricos planos, cujas diferenças nos parâmetros são: coeficiente de resistência média na transferência de calor à condução entre o tudo de aço e o núcleo de concreto de 0.01 $\mathrm{m}^{2} \mathrm{~K} / \mathrm{W}$ e emissividade da face exposta de 0.5 .

Nas Figuras 11 e 12 são indicadas as temperaturas determinadas pelo autor, por meio do software ABAQUS e por Renaud (2004), cujos números indicados nos eixos das abscissas, referem-se às subdivisões da seção transversal, sendo o ponto 6 referente ao tubo de aço e o ponto 1, referente à camada mais interna na seção transversal. 
Tabela 1

Tabela 4

\begin{tabular}{c|c|c|c|c|c|}
\hline \hline \multirow{2}{*}{$\begin{array}{c}\text { Faixas na seção } \\
\text { transversal }\end{array}$} & \multicolumn{5}{|c|}{ Dimensões da seção transversal (b) } \\
\cline { 2 - 6 } & $\mathbf{1 0 0}$ & $\mathbf{1 2 0}$ & $\mathbf{1 4 0}$ & $\mathbf{1 6 0}$ & $\mathbf{2 0 0}$ \\
\hline $0<\mathrm{bi} / \mathrm{b}<0.1$ & 342 & 239 & 153 & 101 & 64 \\
\hline $0.1<\mathrm{bi} / \mathrm{b}<0.2$ & 371 & 272 & 191 & 134 & 84 \\
\hline $0.2<\mathrm{bi} / \mathrm{b}<0.3$ & 430 & 340 & 267 & 208 & 144 \\
\hline $0.3<\mathrm{bi} / \mathrm{b}<0.4$ & 525 & 451 & 389 & 333 & 271 \\
\hline $0.4<\mathrm{bi} / \mathrm{b}<0.5$ & 661 & 626 & $\mathbf{5 8 7}$ & 547 & 510 \\
\hline Tubo de aço & 739 & 717 & 701 & 692 & 684 \\
\hline Concreto & 540 & 474 & 411 & 364 & 307 \\
\hline \multicolumn{7}{|c|}{ Seçăo transversal com tubo quadrado e R30 } \\
\hline
\end{tabular}

\begin{tabular}{|l|c|c|c|c|}
\hline \multirow{2}{*}{$\begin{array}{c}\text { Faixas na seção } \\
\text { transversal }\end{array}$} & \multicolumn{5}{|c|}{ Dimensões da seção transversal $(\mathbf{d})$} \\
\cline { 2 - 5 } & $\mathbf{1 1 4 . 3}$ & $\mathbf{1 4 1 . 3}$ & $\mathbf{1 6 8 . 3}$ & $\mathbf{2 1 9 . 1}$ \\
\hline $0<\mathrm{bi} / \mathrm{b}<0.1$ & 299 & 171 & 94 & $\mathbf{5 2}$ \\
\hline $0.1<\mathrm{bi} / \mathrm{b}<0.2$ & 321 & 202 & 117 & 64 \\
\hline $0.2<\mathrm{bi} / \mathrm{b}<0.3$ & 379 & 279 & 197 & 107 \\
\hline $0.3<\mathrm{bi} / \mathrm{b}<0.4$ & 484 & 400 & 325 & 229 \\
\hline $0.4<\mathrm{bi} / \mathrm{b}<0.5$ & 639 & 590 & 543 & 476 \\
\hline Tubo de aço & 736 & 703 & 686 & 647 \\
\hline Concreto & 422 & 349 & 302 & 180 \\
\hline \multicolumn{5}{|c|}{ Pilar com seção circular e R30 } \\
\hline
\end{tabular}

Tabela 2

\begin{tabular}{|c|c|c|c|c|c|}
\hline \multicolumn{7}{|c|}{ Tabela 2 } \\
\hline \hline $\begin{array}{c}\text { Faixas na seção } \\
\text { transversal }\end{array}$ & $\mathbf{1 0 0}$ & $\mathbf{1 2 0}$ & $\mathbf{1 4 0}$ & $\mathbf{1 6 0}$ & $\mathbf{2 0 0}$ \\
\cline { 2 - 6 } & Dimensões da sec̃ão transversal (b) \\
\hline $0<\mathrm{bi} / \mathrm{b}<0.1$ & 664 & 543 & 443 & 352 & 246 \\
\hline $0.1<\mathrm{bi} / \mathrm{b}<0.2$ & 687 & 573 & 477 & 389 & 286 \\
\hline $0.2<\mathrm{bi} / \mathrm{b}<0.3$ & 732 & 634 & 549 & 467 & 370 \\
\hline $0.3<\mathrm{bi} / \mathrm{b}<0.4$ & 798 & 724 & 663 & 596 & 515 \\
\hline $0.4<\mathrm{bi} / \mathrm{b}<0.5$ & 879 & 849 & 820 & 787 & 748 \\
\hline Tubo de aço & 921 & 898 & 908 & 900 & 894 \\
\hline Concreto & 802 & 735 & 672 & 615 & 537 \\
\hline \multicolumn{7}{|c|}{ Seçắo transversal com tubo quadrado e R60 } \\
\hline
\end{tabular}

\begin{tabular}{|l|c|c|c|c|}
\hline \multicolumn{5}{|c|}{ Tabela 5 } \\
\hline \hline \multirow{2}{*}{$\begin{array}{c}\text { Faixas na seção } \\
\text { transversal }\end{array}$} & \multicolumn{4}{|c|}{ Dimensões da seção transversal (d) } \\
\cline { 2 - 5 } & $\mathbf{1 1 4 . 3}$ & $\mathbf{1 4 1 . 3}$ & $\mathbf{1 6 8 . 3}$ & $\mathbf{2 1 9 . 1}$ \\
\hline $0<\mathrm{bi} / \mathrm{b}<0.1$ & 614 & 493 & 364 & 205 \\
\hline $0.1<\mathrm{bi} / \mathrm{b}<0.2$ & 633 & 517 & 391 & 234 \\
\hline $0.2<\mathrm{bi} / \mathrm{b}<0.3$ & 681 & 582 & 470 & 315 \\
\hline $0.3<\mathrm{bi} / \mathrm{b}<0.4$ & 760 & 684 & 592 & 466 \\
\hline $0.4<\mathrm{bi} / \mathrm{b}<0.5$ & 861 & 826 & 782 & 719 \\
\hline Tubo de aço & 917 & 894 & 888 & 865 \\
\hline Concreto & 707 & 636 & 563 & 378 \\
\hline \multicolumn{5}{|c|}{ Pilar com seçăo circular e R60 } \\
\hline
\end{tabular}

Tabela 3

\begin{tabular}{c|c|c|c|c|c|}
\hline \hline \multirow{2}{*}{$\begin{array}{c}\text { Faixas na seção } \\
\text { transwersal }\end{array}$} & \multicolumn{5}{|c|}{ Dimensões da seção transversal (b) } \\
\cline { 2 - 6 } & $\mathbf{1 0 0}$ & $\mathbf{1 2 0}$ & $\mathbf{1 4 0}$ & $\mathbf{1 6 0}$ & $\mathbf{2 0 0}$ \\
\hline $0<\mathrm{bi} / \mathrm{b}<0.1$ & 834 & 728 & 630 & 535 & 412 \\
\hline $0.1<\mathrm{bi} / \mathrm{b}<0.2$ & 850 & 752 & 661 & 570 & 451 \\
\hline $0.2<\mathrm{bi} / \mathrm{b}<0.3$ & 880 & 799 & 722 & 642 & 535 \\
\hline $0.3<\mathrm{bi} / \mathrm{b}<0.4$ & 922 & 864 & 813 & 755 & 672 \\
\hline $0.4<\mathrm{bi} / \mathrm{b}<0.5$ & 970 & 950 & 928 & 904 & 868 \\
\hline Tubo de aço & 994 & 973 & 987 & 983 & 978 \\
\hline Concreto & 923 & 870 & 815 & 764 & 683 \\
\hline \multicolumn{7}{|c|}{ Seçäo transversal com tubo quadrado e R90 } \\
\hline
\end{tabular}

Tabela 6

\begin{tabular}{|l|c|c|c|c|}
\hline \multirow{2}{*}{$\begin{array}{c}\text { Faixas na seção } \\
\text { transversal }\end{array}$} & \multicolumn{5}{|c|}{ Dimensões da seção transversal (d) } \\
\cline { 2 - 5 } $0<\mathrm{bi} / \mathrm{b}<0.1$ & 793 & 681 & 552 & 370 \\
\hline $0.1<\mathrm{bi} / \mathrm{b}<0.2$ & 806 & 669 & 576 & 399 \\
\hline $0.2<\mathrm{bi} / \mathrm{b}<0.3$ & 841 & 753 & 647 & 478 \\
\hline $0.3<\mathrm{bi} / \mathrm{b}<0.4$ & 895 & 832 & 751 & 623 \\
\hline $0.4<\mathrm{bi} / \mathrm{b}<0.5$ & 956 & 932 & 899 & 842 \\
\hline Tubo de aço & 992 & 971 & 970 & 950 \\
\hline Concreto & 856 & 791 & 721 & 532 \\
\hline \multicolumn{5}{|c|}{ Pilar com seçăo circular e R90 } \\
\hline
\end{tabular}

Figura 9 - Temperatura ao longo da seção transversal $\left({ }^{\circ} \mathrm{C}\right)$

\section{Fonte: autor}

\begin{tabular}{c|c|c|c|c|c|}
\multirow{2}{*}{} & \multicolumn{5}{|c|}{ Dimensões da seção transversal (b) } \\
\cline { 2 - 6 } & $\mathbf{1 0 0}$ & $\mathbf{1 5 0}$ & $\mathbf{2 5 0}$ & $\mathbf{3 5 0}$ & $\mathbf{5 0 0}$ \\
\hline $0<\mathrm{bi} / \mathrm{b}<0.1$ & 345 & 120 & 30 & 20 & 20 \\
\hline $0.1<\mathrm{bi} / \mathrm{b}<0.2$ & 360 & 140 & 45 & 25 & 20 \\
\hline $0.2<\mathrm{bi} / \mathrm{b}<0.3$ & 405 & 210 & 70 & 45 & 25 \\
\hline $0.3<\mathrm{bi} / \mathrm{b}<0.4$ & 470 & 310 & 165 & 100 & 60 \\
\hline $0.4<\mathrm{bi} / \mathrm{b}<0.5$ & 545 & 455 & 365 & 290 & 240 \\
\hline Tubo de aço & 730 & 715 & 695 & 690 & 690 \\
\hline \multicolumn{7}{|c|}{ Seção transwersal com tabo quadrado $\mathrm{R} 30$} \\
\hline
\end{tabular}

\begin{tabular}{|c|c|c|c|c|c|}
\cline { 2 - 6 } & \multicolumn{5}{|c|}{ Dimensões da seç̃o transversal (b) } \\
\cline { 2 - 6 } & $\mathbf{1 0 0}$ & $\mathbf{1 5 0}$ & $\mathbf{2 5 0}$ & $\mathbf{3 5 0}$ & $\mathbf{5 0 0}$ \\
\hline $0<\mathrm{bi} / \mathrm{b}<0.1$ & 670 & 415 & 90 & 50 & 25 \\
\hline $0.1<\mathrm{bi} / \mathrm{b}<0.2$ & 690 & 430 & 130 & 65 & $\mathbf{3 0}$ \\
\hline $0.2<\mathrm{bi} / \mathrm{b}<0.3$ & 710 & 490 & 210 & 115 & 45 \\
\hline $0.3<\mathrm{bi} / \mathrm{b}<0.4$ & 760 & 580 & 360 & 235 & 135 \\
\hline $0.4<\mathrm{bi} / \mathrm{b}<0.5$ & $\mathbf{8 2 0}$ & 700 & 570 & 480 & 125 \\
\hline Tubo de aço & 915 & 895 & 880 & $\mathbf{8 7 5}$ & 870 \\
\hline \multicolumn{7}{|c}{ Seçăo transyersal com tubo quadrado e R60 } \\
\hline
\end{tabular}

\begin{tabular}{|l|c|c|c|c|c|}
\multicolumn{1}{c|}{} & \multicolumn{6}{|c|}{ Dimensões da seção transversal (b) } \\
\cline { 2 - 6 } \multicolumn{1}{c|}{} & $\mathbf{1 0 0}$ & $\mathbf{1 5 0}$ & $\mathbf{2 5 0}$ & $\mathbf{3 5 0}$ & $\mathbf{5 0 0}$ \\
\hline $0<\mathrm{bi} / \mathrm{b}<0.1$ & 860 & 600 & 230 & 100 & 40 \\
\hline $0.1<\mathrm{bi} / \mathrm{b}<0.2$ & 870 & 630 & 270 & 115 & 50 \\
\hline $0.2<\mathrm{bi} / \mathrm{b}<0.3$ & 880 & 670 & 370 & 200 & 90 \\
\hline $0.3<\mathrm{bi} / \mathrm{b}<0.4$ & 910 & 755 & 515 & 365 & 210 \\
\hline $0.4<\mathrm{bi} / \mathrm{b}<0.5$ & 930 & 840 & 715 & 595 & 500 \\
\hline Tubo de aço & 990 & 975 & 960 & 955 & 950 \\
\hline \multicolumn{7}{|c|}{ Seq̧ão transwersal com tubo quadrado e R90 } \\
\hline
\end{tabular}

\begin{tabular}{|l|c|c|c|c|c|}
\cline { 2 - 6 } \multicolumn{1}{c|}{} & \multicolumn{5}{c|}{ Dimensões da seção transversal (b) } \\
\cline { 2 - 6 } \multicolumn{1}{c|}{} & $\mathbf{1 0 0}$ & $\mathbf{1 5 0}$ & $\mathbf{2 5 0}$ & $\mathbf{3 5 0}$ & $\mathbf{5 0 0}$ \\
\hline $0<\mathrm{bi} / \mathrm{b}<0.1$ & 975 & 745 & 365 & 150 & 60 \\
\hline $0.1<\mathrm{bi} / \mathrm{b}<0.2$ & 970 & 770 & 410 & 195 & 90 \\
\hline $0.2<\mathrm{bi} / \mathrm{b}<0.3$ & 990 & 860 & 490 & 295 & 145 \\
\hline $0.3<\mathrm{bi} / \mathrm{b}<0.4$ & 1000 & 860 & 490 & 295 & 145 \\
\hline $0.4<\mathrm{bi} / \mathrm{b}<0.5$ & 1015 & 935 & 805 & 695 & 610 \\
\hline Tubo de aço & 1040 & 1030 & 1015 & 1010 & 1005 \\
\hline \multicolumn{7}{|c|}{ Seção transversal com tubo quadrado e R.120 } \\
\hline
\end{tabular}




\begin{tabular}{|c|c|c|c|c|c|}
\multirow{2}{*}{} & \multicolumn{5}{|c|}{ Dimensões da seção transversal (d) } \\
\cline { 2 - 6 } & $\mathbf{1 0 0}$ & $\mathbf{1 5 0}$ & $\mathbf{2 5 0}$ & $\mathbf{3 5 0}$ & $\mathbf{5 0 0}$ \\
\hline $0<\mathrm{bi} / \mathrm{b}<0.1$ & 390 & 145 & 40 & 20 & 20 \\
\hline $0.1<\mathrm{bi} / \mathrm{b}<0.2$ & 400 & 180 & 60 & 20 & 20 \\
\hline $0.2<\mathrm{bi} / \mathrm{b}<0.3$ & 440 & 255 & 95 & 50 & 25 \\
\hline $0.3<\mathrm{bi} / \mathrm{b}<0.4$ & 500 & 365 & 205 & 120 & 60 \\
\hline $0.4<\mathrm{bi} / \mathrm{b}<0.5$ & 600 & 520 & 430 & 365 & 290 \\
\hline Tubo de aço & 740 & 730 & 720 & 715 & 713 \\
\hline \multicolumn{6}{|c|}{ Pilar com secãa circular \& R30 } \\
\hline
\end{tabular}

\begin{tabular}{|l|c|c|c|c|c|}
\multicolumn{1}{c|}{} & \multicolumn{6}{c|}{ Dimensões da seção transversal (d) } \\
\cline { 2 - 6 } \multicolumn{1}{c|}{} & $\mathbf{1 0 0}$ & $\mathbf{1 5 0}$ & $\mathbf{2 5 0}$ & $\mathbf{3 5 0}$ & $\mathbf{5 0 0}$ \\
\hline $0<\mathrm{bi} / \mathrm{b}<0.1$ & 890 & 655 & $\mathbf{3 0 0}$ & 100 & 40 \\
\hline $0.1<\mathrm{bi} / \mathrm{b}<0.2$ & 900 & 685 & $\mathbf{3 3 5}$ & 150 & 60 \\
\hline $0.2<\mathbf{b i} / \mathrm{b}<0.3$ & 910 & 730 & 405 & 240 & 110 \\
\hline $0.3<\mathrm{bi} / \mathrm{b}<0.4$ & 930 & 805 & 550 & 405 & 235 \\
\hline $0.4<\mathrm{bi} / \mathrm{b}<0.5$ & 960 & 872 & 760 & 675 & 580 \\
\hline Tubo de aço & 995 & 985 & 975 & 968 & 965 \\
\hline \multicolumn{7}{|c|}{ Pi.lar com seçăo circular e R90 } \\
\hline
\end{tabular}

\begin{tabular}{|c|c|c|c|c|c|}
\cline { 2 - 6 } & \multicolumn{5}{|c|}{ Dimens̃es da seção transversal (d) } \\
\cline { 2 - 6 } & $\mathbf{1 0 0}$ & $\mathbf{1 5 0}$ & $\mathbf{2 5 0}$ & $\mathbf{3 5 0}$ & $\mathbf{5 0 0}$ \\
\hline $0<\mathrm{bi} / \mathrm{b}<0.1$ & 700 & 445 & 130 & 60 & 25 \\
\hline $0.1<\mathrm{bi} / \mathrm{b}<0.2$ & 730 & 480 & 180 & 75 & 30 \\
\hline $0.2<\mathrm{bi} / \mathrm{b}<0.3$ & 745 & 540 & 270 & 135 & 60 \\
\hline $0.3<\mathrm{bi} / \mathrm{b}<0.4$ & 785 & 625 & 400 & 275 & 160 \\
\hline $0.4<\mathrm{bi} / \mathrm{b}<0.5$ & 845 & 755 & 640 & 555 & 480 \\
\hline Tubo de aço & 920 & 905 & 895 & 885 & 880 \\
\hline \multicolumn{7}{|c|}{ Pilar com seçăa circular e R60 } \\
\hline
\end{tabular}

\begin{tabular}{|l|c|c|c|c|c|}
\cline { 2 - 6 } \multicolumn{1}{c|}{} & \multicolumn{6}{c|}{ Dimensões da seção transversal (d) } \\
\cline { 2 - 6 } \multicolumn{1}{c|}{} & $\mathbf{1 0 0}$ & $\mathbf{1 5 0}$ & $\mathbf{2 5 0}$ & $\mathbf{3 5 0}$ & $\mathbf{5 0 0}$ \\
\hline $0<\mathrm{bi} / \mathrm{b}<0.1$ & 975 & 805 & 405 & 188 & 60 \\
\hline $0.1<\mathrm{bi} / \mathrm{b}<0.2$ & 980 & 810 & 460 & 233 & 90 \\
\hline $0.2<\mathrm{bi} / \mathrm{b}<0.3$ & 990 & 840 & 537 & 327 & 170 \\
\hline $0.3<\mathrm{bi} / \mathrm{b}<0.4$ & 1000 & 880 & 670 & 495 & $\mathbf{3 4 0}$ \\
\hline $0.4<\mathrm{bi} / \mathrm{b}<0.5$ & 1015 & 965 & 850 & 760 & 665 \\
\hline Tubo de aço & 1042 & 1035 & 1025 & 1020 & 1015 \\
\hline \multicolumn{7}{|c|}{ Pilar com seçâio circular E R120 } \\
\hline
\end{tabular}

Figura 10 - Temperatura ao longo da seção transversal $\left({ }^{\circ} \mathrm{C}\right)$ Fonte: Renaud (2004)
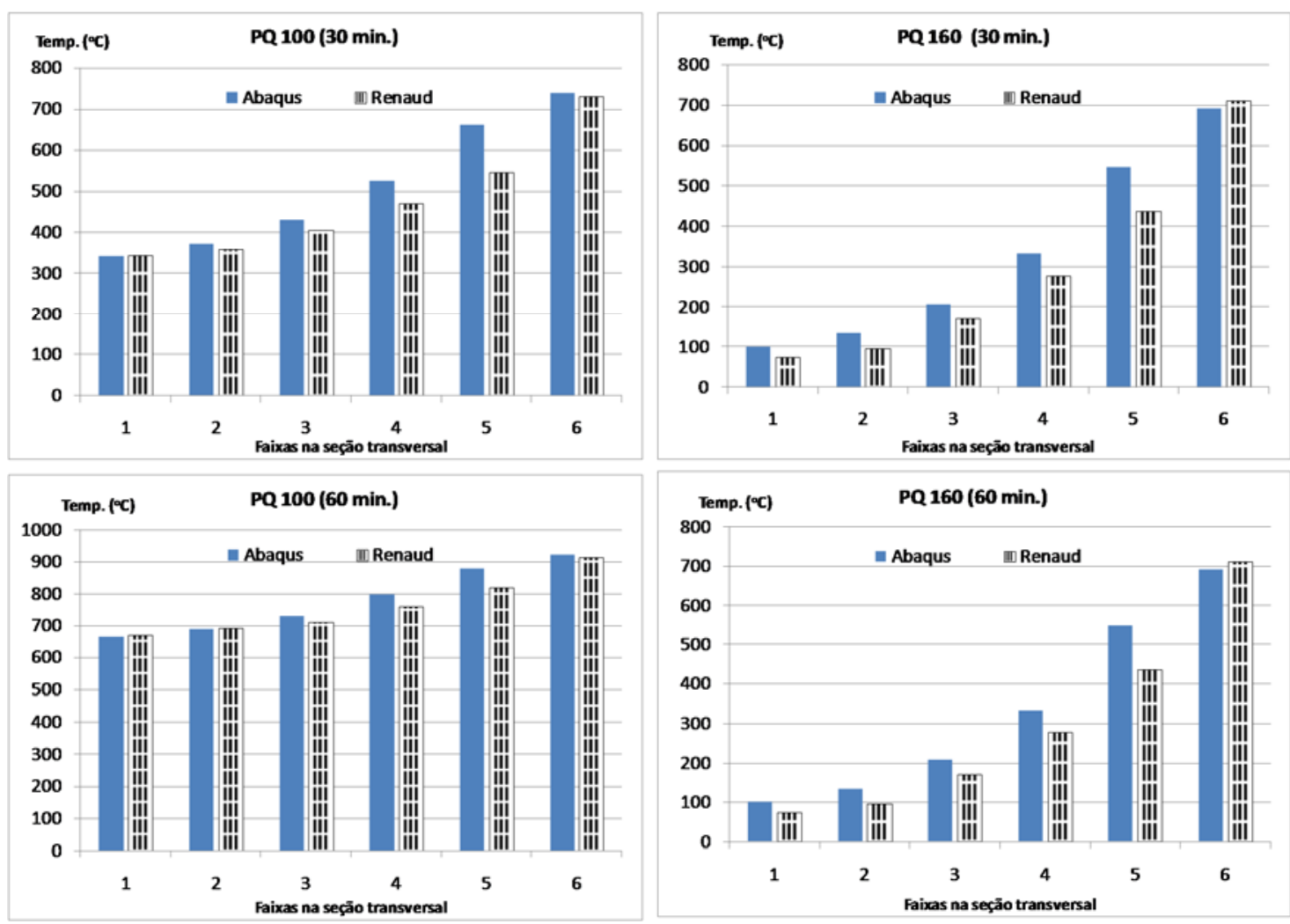

Figura 11 - Temperaturas ao longo da seção transversal quadrada 


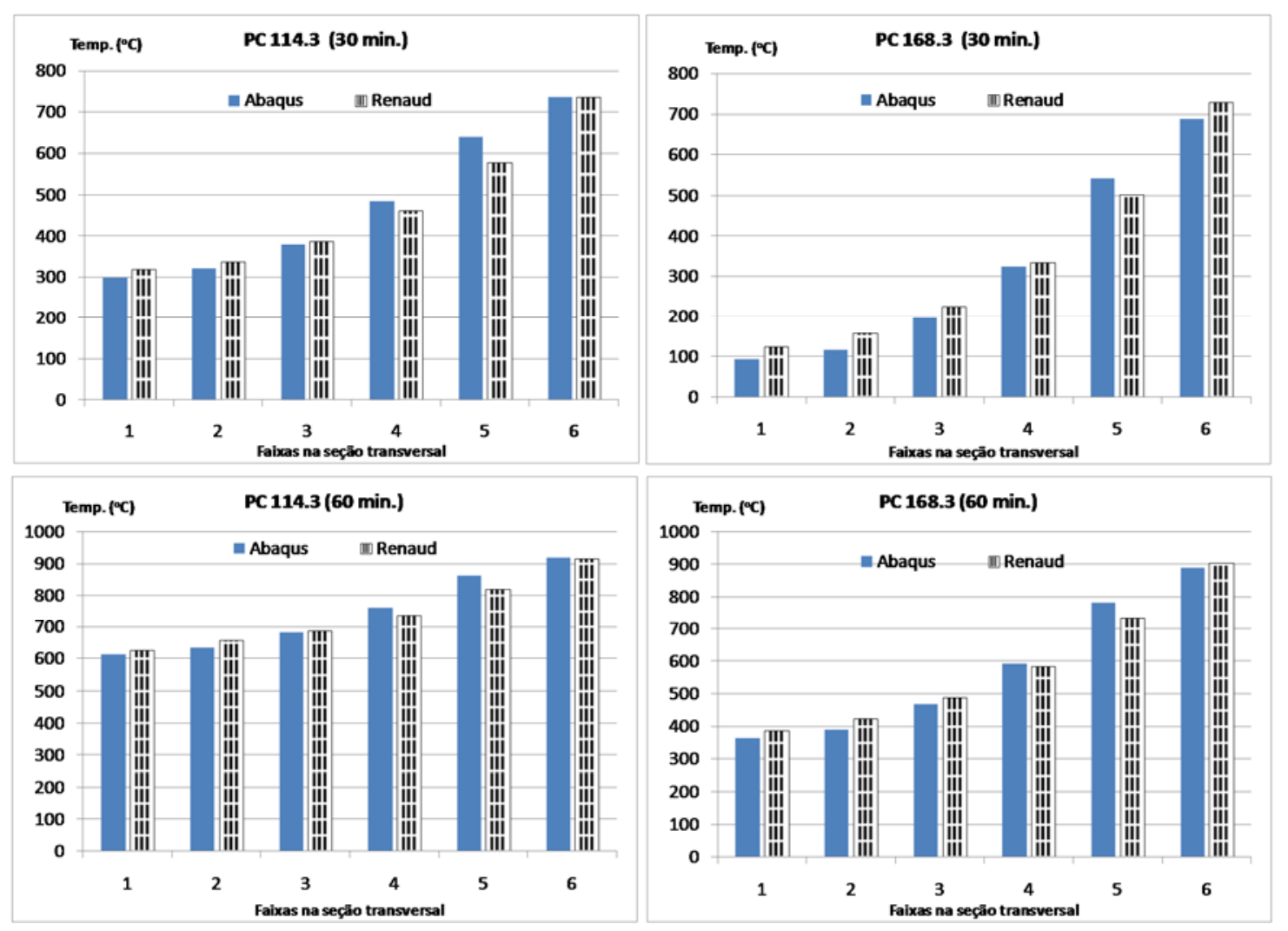

Figura 12 - Temperaturas ao longo da seção transversal circular

\subsection{Dimensionamento em situação de incêndio}

Para a análise comparativa dos processos simplificados foram elaborados modelos numéricos tridimensionais por meio do software $A B A Q U S$, cujos resultados de alguns exemplares, seguem indicados na Tabela 2.

Tabela 2 - Resultados dos modelos numéricos tridimensionais (análise termomecânica)

\begin{tabular}{|c|c|c|}
\hline \multicolumn{3}{|c|}{ Resultados modelos numéricos } \\
\hline Exemplar & Normal $(\mathrm{kN})$ & TRF (min.) \\
\hline PC-114.3-4 & 142 & 48,9 \\
\hline PC-168.3-6.4 & 522 & 36,8 \\
\hline PC-168.3-6.4 & 618 & 35,2 \\
\hline PQ-100-5.2 & 148 & 52 \\
\hline PQ-140-5.6 & 442 & 38,2 \\
\hline PQ-140-5.6 & 477 & 37.8 \\
\hline
\end{tabular}

Nas Figuras 13 e 14 é apresentado o gráfico referente à contração axial do pilar e taxa de contração axial em função do tempo, de alguns dos exemplares mencionados, cujo critério de falha adotado é descrito na EN 1363 (1999). 

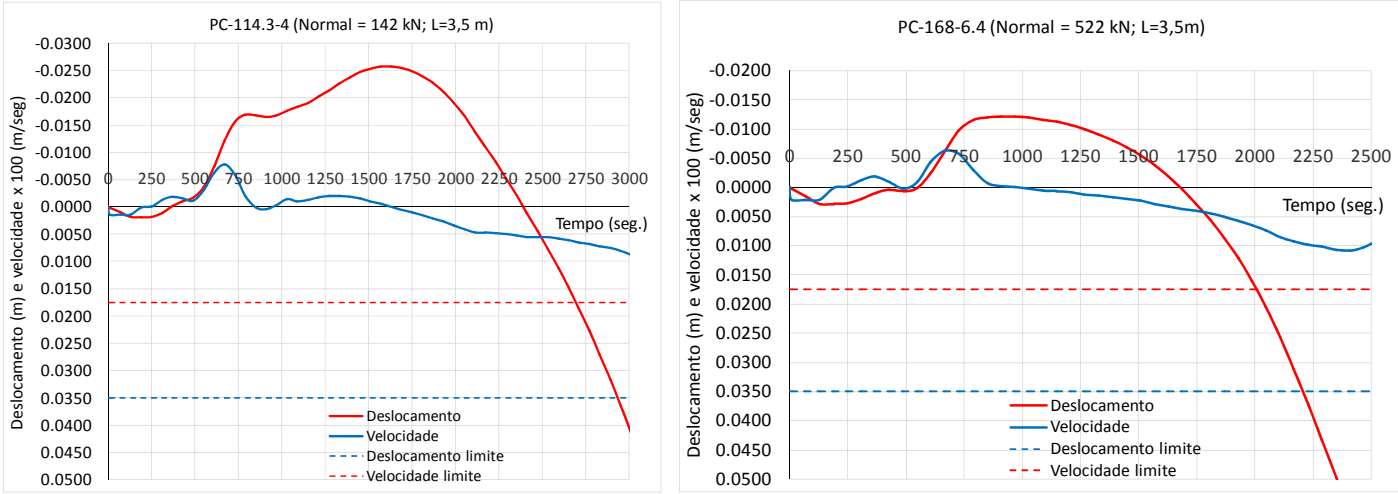

Figura 13 - Critério de falha pilar de seção circular
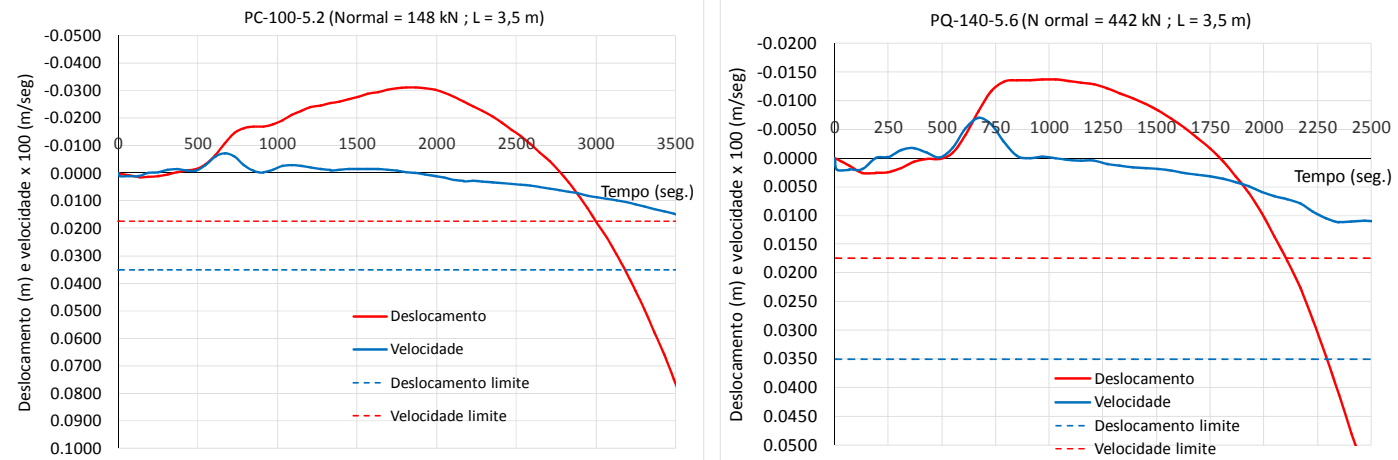

Figura 14 - Critério de falha pilar de seção quadrada

Através dos processos simplificados, foi determinada a normal última de cada exemplar, considerando comprimentos de flambagem distintos e temperaturas de exposição ao fogo de 30 e 60 minutos.

Na Figura 15, o procedimento 1 , considera às temperaturas tomadas nas tabelas da Figura 9, definidas por meio do software ABAQUS e dimensionamento pelo método geral; o procedimento 2 , às temperaturas tomadas das tabelas elaboradas por Renaud (Figura 10) e dimensionamento pelo método geral; procedimento 3, às temperaturas definidas de forma simplificada pelas equações descritas em 2.5.2. e dimensionamento pelo método geral; procedimento 4 , às temperaturas obtidas por meio do software ABAQUS e dimensionamento pelo método geral, considerando a curva de dimensionamento "a". 


\begin{tabular}{|c|c|c|c|c|c|c|c|c|c|}
\hline \multicolumn{5}{|c|}{ Pilar com seção quadrada, $b=100 \mathrm{~mm}$} & \multicolumn{5}{|c|}{ Pilar com seção quadrada, $b=100 \mathrm{~mm}$} \\
\hline \multirow{2}{*}{$\begin{array}{l}\text { Procedimento } \\
\text { utilizado }\end{array}$} & \multicolumn{4}{|c|}{ Comprimento de flambagem (mm) } & \multirow{2}{*}{$\begin{array}{l}\text { Procedimento } \\
\text { utilizado }\end{array}$} & \multicolumn{4}{|c|}{ Comprimento de flambagem (mm) } \\
\hline & 1250 & 1500 & 1750 & 2100 & & 1250 & 1500 & 1750 & 2100 \\
\hline 1 & 185 & 160.8 & 137.7 & 109.9 & 1 & 65 & 58.9 & 52.7 & 44.2 \\
\hline 2 & 200.5 & 173.2 & 147.6 & 117.1 & 2 & 70.5 & 63.5 & 56.4 & 46.9 \\
\hline 3 & 195.3 & 170.4 & 146.5 & 117.3 & 3 & 57.3 & 52.6 & 47.7 & 40.9 \\
\hline 4 & 218.6 & 196.3 & 169.8 & 133.8 & 4 & 73.7 & 69.3 & 63.9 & 54.6 \\
\hline \multicolumn{5}{|c|}{ Tempo de exposição ao fogo de $30 \mathrm{~min}$. (Normal última em kN) } & \multicolumn{5}{|c|}{ Tempo de exposição ao fogo de $60 \mathrm{~min}$. (Normal última em $\mathrm{kN}$ ) } \\
\hline \multicolumn{5}{|c|}{ Pilar com seção quadrada, $b=140 \mathrm{~mm}$} & \multicolumn{5}{|c|}{ Pilar com seção quadrada, $b=140 \mathrm{~mm}$} \\
\hline \multirow{2}{*}{$\begin{array}{c}\text { Procedimento } \\
\text { utilizado }\end{array}$} & \multicolumn{4}{|c|}{ Comprimento de flambagem (mm) } & Procedimento & \multicolumn{4}{|c|}{ Comprimento de flambagem (mm) } \\
\hline & 1250 & 1500 & 1750 & 2100 & utilizado & 1250 & 1500 & 1750 & 2100 \\
\hline 1 & 480.3 & 439.4 & 396.7 & 337.9 & 1 & 195.7 & 179.7 & 162.9 & 139.5 \\
\hline 2 & 495.8 & 453.2 & 409 & 347.8 & 2 & 222.5 & 202.8 & 182.4 & 154.5 \\
\hline 3 & 521.1 & 482.5 & 441.8 & 384 & 3 & 174.4 & 162 & 148.9 & 130.1 \\
\hline 4 & 538.9 & 511.5 & 476.6 & 415.7 & 4 & 218.7 & 208.2 & 194.8 & 171.4 \\
\hline \multicolumn{5}{|c|}{ Tempo de exposição ao fogo de 30 min. (Normal última em kN) } & \multicolumn{5}{|c|}{ Tempo de exposição ao fogo de 60 min. (Normal última em kN) } \\
\hline \multicolumn{5}{|c|}{ Pilar com seção quadrada, $b=200 \mathrm{~mm}$} & \multicolumn{5}{|c|}{ Pilar com seção quadrada, $b=200 \mathrm{~mm}$} \\
\hline \multirow{2}{*}{$\begin{array}{c}\text { Proced imento } \\
\text { utilizado }\end{array}$} & \multicolumn{4}{|c|}{ Comprimento de flambagem (mm) } & Procedimento & \multicolumn{4}{|c|}{ Comprimento de flambagem (mm) } \\
\hline & 1250 & 1500 & 1750 & 2100 & utilizado & 1250 & 1500 & 1750 & 2100 \\
\hline 1 & 1208 & 1152.4 & 1094.2 & 1008.2 & 1 & 589.8 & 554.6 & 517.4 & 462.5 \\
\hline 2 & 1170.4 & 1112.4 & 1051.5 & 961.6 & 2 & 663.3 & 621.9 & 578.2 & 514.5 \\
\hline 3 & 1257.5 & 1204 & 1148.3 & 1066.2 & 3 & 523.1 & 493.7 & 462.7 & 417.2 \\
\hline 4 & 11278 & 1249.5 & 1216.9 & 1162 & 4 & 638.4 & 618.6 & 594.9 & 552.7 \\
\hline \multicolumn{5}{|c|}{ Tempo de exposição ao fogo de 30 min. (Normal última em kN) } & \multicolumn{5}{|c|}{ Tempo de exposição ao fogo de $60 \mathrm{~min}$. (Normal última em kN) } \\
\hline \multicolumn{5}{|c|}{ Pilar com seção circular, $d=114.3 \mathrm{~mm}$} & \multicolumn{5}{|c|}{ Pilar com seção circular, $d=114.3 \mathrm{~mm}$} \\
\hline & & imento & mbagen & & Procedimento & & mento & mbage & \\
\hline utilizado & 1250 & 1500 & 1750 & 2100 & utilizado & 1250 & 1500 & 1750 & 2100 \\
\hline 1 & 163.6 & 138.1 & 115.4 & 89.7 & 1 & 63.2 & 55.2 & 47.5 & 38 \\
\hline 2 & 168.4 & 141.8 & 118.2 & 91.7 & 2 & 65.3 & 56.8 & 48.6 & 38.8 \\
\hline 3 & 114.6 & 93.7 & 79.1 & 64.8 & 3 & 45 & 37.7 & 32.5 & 27.3 \\
\hline 4 & 197.7 & 170.2 & 141.5 & 107.5 & 4 & 74.4 & 67.2 & 58.5 & 46.4 \\
\hline Tempo de & ição ao & $30 \mathrm{~min}$. & nal últim & $\mathrm{kN})$ & Tempo de & ção ao & $60 \mathrm{~min}$. & nal últin & \\
\hline & com sec & rcular, & $88.3 \mathrm{~mm}$ & & & om seg & cular, & $58.3 \mathrm{mr}$ & \\
\hline Procedimento & & imento & mbagen & & Procedimento & & mento & mbage & \\
\hline utilizado & 1250 & 1500 & 1750 & 2100 & utilizado & 1250 & 1500 & 1750 & 2100 \\
\hline 1 & 616.2 & 570.3 & 522.1 & 453.4 & 1 & 265.6 & 242.8 & 219.1 & 186.3 \\
\hline 2 & 547.2 & 502.4 & 455.6 & 390.3 & 2 & 264.9 & 241.3 & 216.9 & 183.5 \\
\hline 3 & 389.1 & 342.1 & 298.9 & 250.3 & 3 & 164.3 & 142.5 & 124.4 & 105 \\
\hline 4 & 681.6 & 653 & 617.4 & 553.6 & 4 & 298.2 & 282.9 & 263.3 & 229.3 \\
\hline Tempo de & ição ao & $30 \mathrm{~min}$. & nal últim & $\mathrm{kN})$ & Tempo d & ção ao & $60 \mathrm{~min}$. & nal últin & \\
\hline
\end{tabular}

Figura 15 - Normal última dos pilares de seção quadrada e circular

Seguem na Figura 16, os respectivos gráficos comparativos para alguns dos exemplares indicados na Figura 1.

Conforme se observa, há uma razoável concordância entre os procedimentos 1 e 2, já o procedimento 3 resulta em diferenças mais expressivas para os pilares com seção circular, contudo, com valores conservadores. O procedimento 4, que adota a curva "a" de dimensionamento, resultam em valores significativamente maiores que os demais, que adotam a curva "c". 

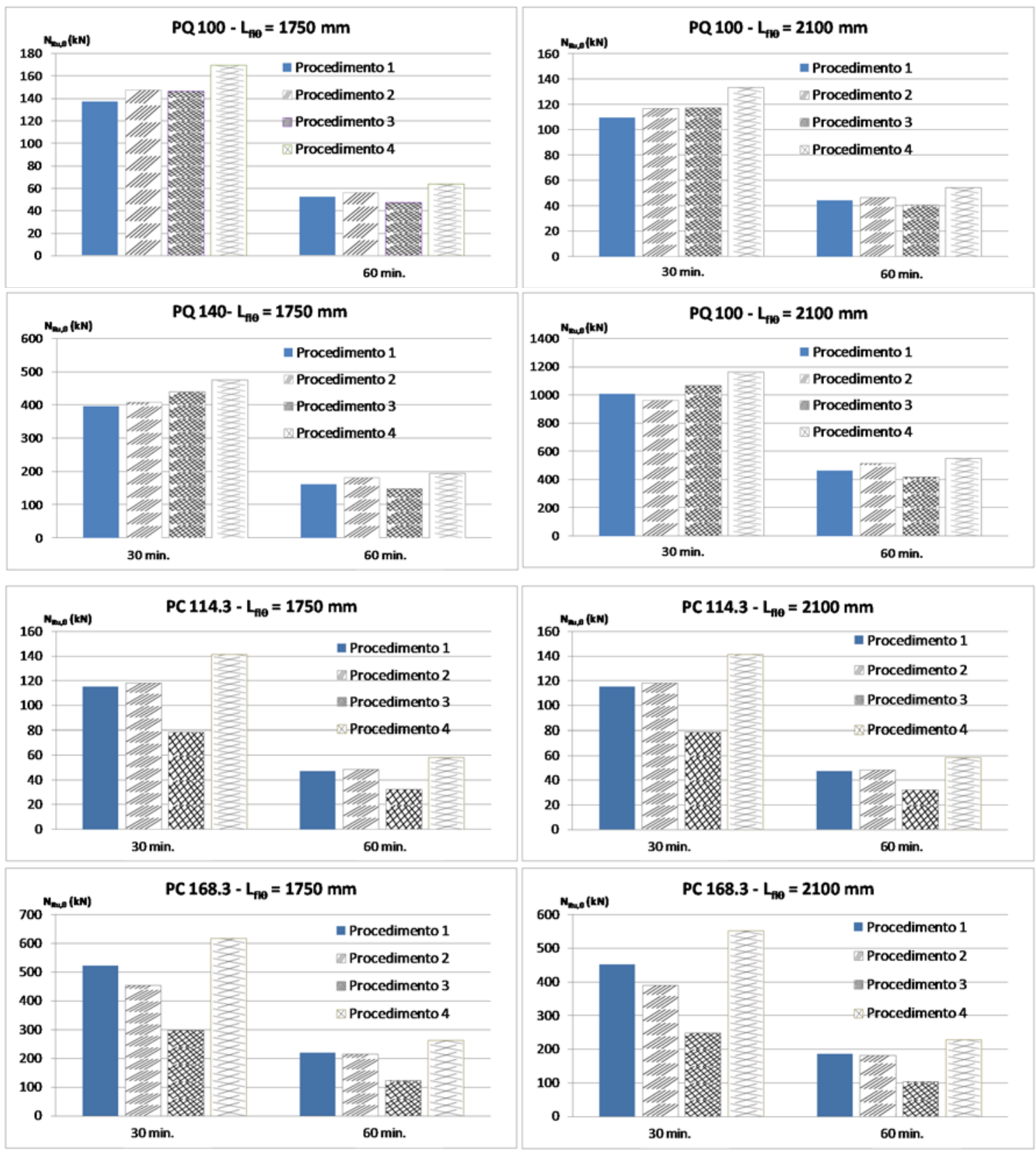

Figura 16 - Normal última dos pilares de seção quadrada e circular

\section{Conclusões}

Com o estudo realizado pode-se observar que o processo analítico simplificado descrito no Eurocode 4 pode ser utilizado para dimensionamento de pilares mistos compostos por tubos de aço de menor dimensão, fora do limite de aplicação indicado pela norma. Os processos simplificados fornecem resultados satisfatórios e normalmente conservadores, podendo ser utilizadas as tabelas de temperaturas propostas nesse trabalho, Figura 9 ou, as tabelas indicadas por Renaud (2004), Figura 10. 
Os resultados dos modelos numéricos, elaborados no presente trabalho, resultam em tempos de resistência ao fogo sempre maiores que os obtidos pelos processos simplificados.

Deve ser utilizada a curva de dimensionamento "c" indicada no Eurocode 3, exceto para o processo indicado em Espinós (2012), que utiliza a curva "a" e cujas respostas mostraram ser sempre conservadoras.

Todos os estudos foram realizados considerando a força axial aplicada ao pilar de forma centrada, portanto, para verificar a aplicação dos processos simplificados em pilares com força axial excêntrica, um estudo semelhante ao apresentado deve ser realizado, considerando a elaboração de modelos numéricos com diferentes excentricidades de aplicação da força axial e com pilares de diferentes comprimentos longitudinais.

\section{Agradecimentos}

Gostaria de agradecer a meu orientador, Dr. Armando Lopes Moreno Junior, pelo incentivo e dedicação na orientação e à Universidade Católica de Santos, pelo suporte que tenho recebido.

\section{Referências bibliográficas}

ARIBERT, J. M. et al, 2008, "Simple fire design for composite hollow section columns". Structures \& Buildings 161:325-336.

ASSOCIAÇÃO BRASILEIRA DE NORMAS TÉCNICAS - "Exigências de resistência aofogo de elementos construtivos de edificações - Procedimento" - NBR 14432: 2001.

ASSOCIAÇÃO BRASILEIRA DE NORMAS TÉCNICAS - "Ações e Segurança nas Estruturas" - NBR 8681: 2004.

EUROPEAN COMMITTEE FOR STANDARDIZATION. EUROCODE 4: "Design of composite steel and concrete structures - Part 1.2: General Rules - Structural fire design" - EN 1994-1-2, 2005.

EUROPEAN COMMITTEE FOR STANDARDIZATION. EUROCODE 3: "Design of steel structures Part 1.1: General rules and rules for buildings" - EN 1993-1-1, 2005.

European Committee for Standardization (CEN): "Fire resistance tests - Part 1: General requerements" - pr EN 1363-1. Brussels, 1999.

ESPINÓS CAPILLA, A., 2012. "Numerical analysis of the fire resistance of circular and elliptical slender concrete filled tubular columns". Doctoral thesis. Universitat Politècnica de València, Spain. 
HAGER I.; KRZEMIEN, K. - "An overview of concrete modulus of elasticity evolution with temperature and comments to European code provisions" - International Fire Safety Symposium, Coimbra, Portugal, Abril, 2015.

INTERNATIONAL STANDARD (1999). Fire-resistance tests - Elements of building construct: General requirements. ISO 834-1:1999.

RENAUD, C. et al, CTICN, 2004. Research Project 15Q. Report reference INSI - 04/75b - CR/PB, France.

RIGAZZO, A. O. et al., 2006. "Dimensionamento de pilares tubulares de aço preenchidos com concreto em situação de incêndio". São Carlos. Dissertação de Mestrado - Escola de Engenharia de São Carlos - Universidade de São Paulo, 2007.

RODRIGUES, M. F.; MORENO Jr., A. L. - " Temperature field of concrete-filled steel tubular columns in fire" - .REM - Revista Escola de Minas, Ouro Preto, 70(2), 137-147, 2017.

RODRIGUES, J. P., EUROPEAN PROJECT FRISCC - "Finite Element Modeling of Innovative Concrete-Filled Tubular Columns Under Roon and Elevanted Temperatures", 2012.

VARGAS, M. R., SILVA, V. P. (2003). Resistência do Fogo das Estruturas de Aço, Instituto Brasileiro de Siderurgia - IBS / Centro Brasileiro da Construção em Aço - CBCA, Rio de Janeiro, 76p.

WANG, Y. C., 1997. "Some considerations in the design of unprotected concrete-filled steel tubular columns under fire conditions". Journal of Constructional Steel Research 44(3):203-223. 